\title{
Bilateral Emphysematous Pyelonephritis and Emphysematous Cystitis with Autosomal- Dominant Polycystic Kidney Disease: Is Conservative Management Justified?
}

\author{
Narayanaswamy Arun Abdullatif Hussain Madan Mohan Kapoor Fawzi Abul
}

Departments of Surgery (Urology Unit) and Nephrology, Al-Amiri Hospital, Kuwait

\section{Key Words}

Emphysematous pyelonephritis • Emphysematous cystitis · Autosomal-dominant polycystic kidney disease • Conservative management

\begin{abstract}
Objective: To report a case of bilateral emphysematous pyelonephritis (EPN) and emphysematous cystitis in a 64-yearold diabetic male with autosomal-dominant polycystic kidney disease (ADPKD). Case Presentation and Intervention: A 64-year-old diabetic male presented with worsening of renal function and fluid overload. Diagnosis was confirmed by computerized tomography (CT scan) and conservative management with broad-spectrum antibiotics was instituted. There was good clinical response and repeated CT scan showed complete resolution. Conclusion: This case shows that conservative management is an acceptable alternative to surgery in EPN occurring with ADPKD. However, it is recommended that patients should be closely monitored, both clinically and radiologically, and percutaneous catheter drainage or surgical intervention carried out whenever deemed necessary.

Copyright $\odot 2007$ S. Karger AG, Basel
\end{abstract}

\section{Introduction}

Emphysematous pyelonephritis (EPN) is a rare, necrotizing form of acute renal infection that results in the presence of gas in the renal parenchyma, the perinephric tissue, or collecting system [1]. Almost $60-70 \%$ of cases are associated with uncontrolled diabetes mellitus, with or without obstructive uropathy and superimposed infection with gas forming organisms [2]. Facultative anaerobes that are able to ferment glucose to lactate and $\mathrm{CO}_{2}$ such as Escherichia coli, Proteus, Pseudomonas, Klebsiella, Aerobacter are possible causative organisms, with $E$. coli being the commonest [2-4]. We report a case of bilateral EPN and emphysematous cystitis occurring with autosomal-dominant polycystic kidney disease (ADPKD).

\section{Case Report}

A 64-year-old male, a known case of ADPKD and renal failure, presented with worsening of renal function and fluid overload. He had a history of insulin-dependent diabetes mellitus, hypertension, ischemic heart disease, and multiple cerebrovascular accidents, and had previously undergone coronary artery bypass grafting.

Physical examination revealed an ill-appearing man with normal consciousness, vital signs, and blood pressure. Auscultation

Dr. Narayanaswamy Arun

Urology Unit, Ward 4, Al-Amiri Hospital

PO Box 4077

13041 Safat (Kuwait)

Tel. +965 6299 701, Fax +965 2424 135, E-Mail nandarun@hotmail.com 
Fig. 1. CT scan showing numerous cysts of variable sizes, marked parenchymal thinning and poor contrast excretion. Multiple pockets of air density pattern are seen bilaterally in the renal parenchyma and collecting systems. Urinary bladder wall and lumen show air streaks.

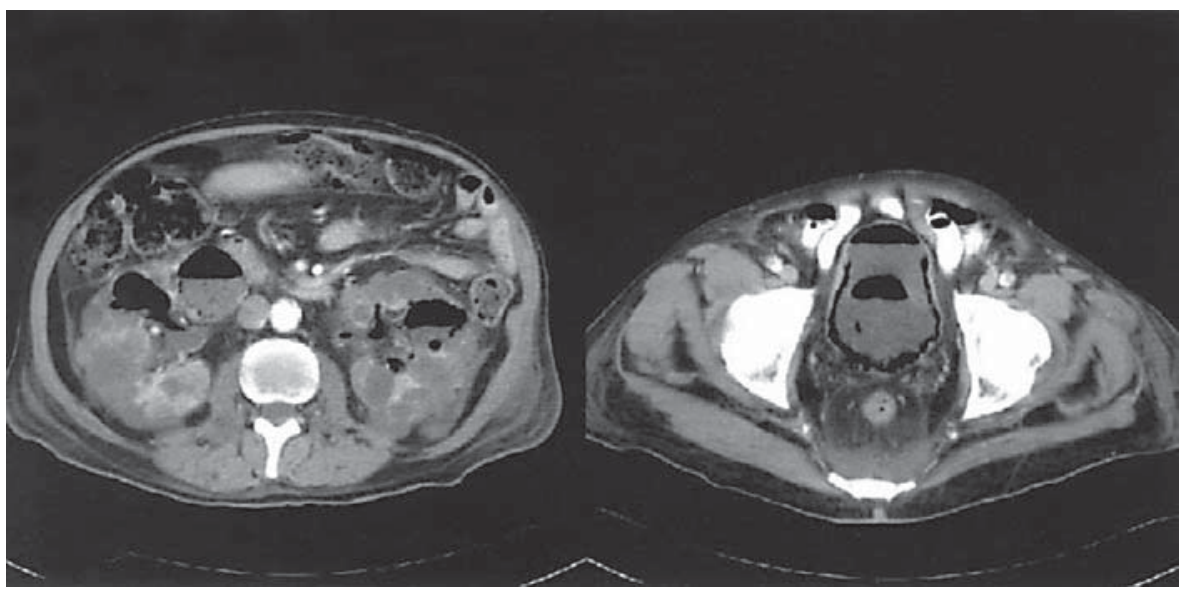

Fig. 2. CT scan after 4 weeks showing complete resolution of the air pockets within the kidneys and the bladder.

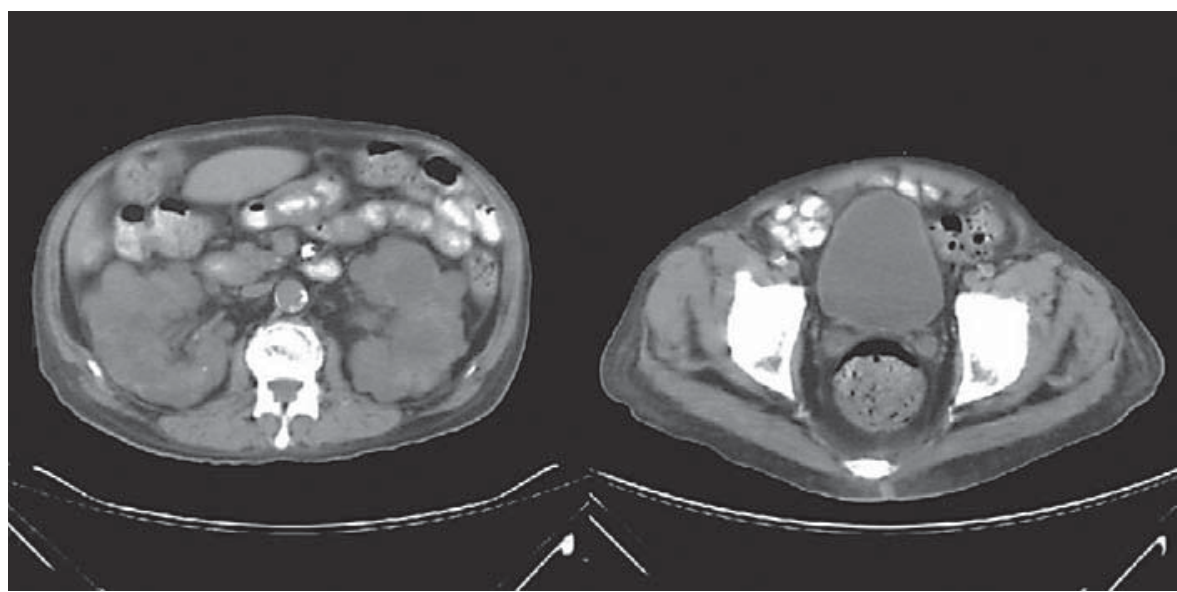

of the chest revealed bilateral crepitations. Examination of the abdomen revealed bilateral palpable kidneys, which were not tender.

Laboratory evaluation showed normal white cell and platelet count, hemoglobin $78 \mathrm{~g} / \mathrm{l}$ (normal 130-170), serum creatinine $522 \mu \mathrm{mol} / \mathrm{l}$ (normal 62-115), blood glucose $31 \mathrm{mmol} / \mathrm{l}$ (normal 3.9-6.1) and marked pyuria. Ultrasonography showed bilateral polycystic kidneys and no perinephric collection. Urine was sampled for bacterial culture and management was instituted with control of blood glucose, maintenance of fluid balance with dialysis, and empirical antibiotics (cefotaxime). Urine culture showed no growth and following dialysis transient hematuria was observed. Seven days after admission he developed fever, turbid urine, neutrophilic leukocytosis, and elevated blood glucose. $\mathrm{He}$ still had no loin pain or tenderness and was hemodynamically stable. Computerized tomography (CT scan) showed bilateral polycystic kidney with multiple pockets of air in both renal parenchyma and collecting systems (fig. 1); there was no air in the perinephric region and the urinary bladder wall and lumen showed air streaks. In view of his hemodynamic stability, absence of loin tenderness, and multiple medical risk factors, it was decided to continue conservative management with antibiotics. Repeated urine culture grew E. coli and Staphylococcus aureus, and antibiotics were changed to tazocin and vancomycin according to the sensitivity test. He showed good clinical response 4 days later, when his urine became clear and serum creatinine was stable at $430 \mu \mathrm{mol} / \mathrm{l}$ without dialysis. Fever subsided after 1 week and repeated CT scan showed marked reduction in the air in the right kidney and complete resolution of the air in the left kidney and bladder. Antibiotics were continued and CT scan was repeated after 4 weeks, which showed complete resolution of the air in both the kidneys, and in the bladder (fig. 2). He was discharged 42 days after admission on oral antibiotics and was doing well at 3 months' follow-up.

\section{Discussion}

EPN occurring with ADPKD is very rare with only 4 cases being previously reported [5-8]. Although concurrent EPN and emphysematous cystitis has been reported before [9], to our knowledge, this is the first case to document bilateral EPN and emphysematous cystitis occurring with ADPKD. The etiology of EPN in ADPKD is not clear. It has been suggested that the preexisting damaged 
renal tissue and impaired vascular supply might be a precipitating factor, as certain bacteria can use the necrotic tissue as a substrate for gas formation [6]. The hyperglycemia in septic diabetic patients and impeded host response favors the bacterial overgrowth [3]. Ureteral instrumentation leading to seeding of bacteria into cysts has also been reported to be the cause in 1 case [5].

Mortality reportedly occurs in $20 \%$ of unilateral EPN and $50 \%$ of bilateral EPN, so appropriate management is essential [3]. Conservative medical treatment was preferred in early reports of EPN [1]. Later series showed a better survival with early aggressive surgical treatment (nephrectomy) in comparison to medical treatment (75 vs. 29\%) [5] and decreased antibiotic perfusion was suggested to be the cause of failure of medical treatment [2]. Prompt diagnosis and surgical intervention was therefore suggested to be the most effective way of managing EPN $[3,5]$. However, in patients with bilateral disease and in patients with associated medical risk factors the morbidity associated with this approach was higher. An alternative medical approach with broad-spectrum antibiotics, maintenance of fluid balance, control of blood glucose and intensive circulatory support was suggested [2, 4]. Factors stated to be essential for success of medical management include early diagnosis, no obstructive uropathy, and adequate perfusion visualized on renal flow scan. Persistence of localized gas within the renal parenchyma and/or the pyelocalyceal system is not per se a discouraging sign [1]. Huang and Tseng [10] suggested antibiotic treatment combined with percutaneous catheter drainage, wherever necessary, in patients with localized
EPN and in patients with extensive EPN with a more benign manifestation (less than two of the following four risk factors including thrombocytopenia, acute renal function impairment, disturbances of consciousness, and shock).

Treatment in the 4 reported cases of EPN with ADPKD included bilateral nephrectomy in 1, unilateral nephrectomy in 1, conservative management in 1 , and not defined in 1 [5-8]. Our patient was managed conservatively despite extensive disease and outcome was good, probably because of early diagnosis, presence of only one risk factor, and appropriate antibiotic therapy. The type and duration of antibiotic therapy cannot be generalized due to limited experience. It appears that in the early phase of EPN, and when the patient is hemodynamically stable, conservative management can be successful. However, the patient should be closely monitored, both clinically and radiologically, and percutaneous catheter drainage or surgical intervention carried out whenever necessary.

\section{Conclusion}

This case demonstrates that conservative management along with close monitoring can be a successful modality in the treatment of EPN occurring with ADPKD despite extensive disease, and is an acceptable alternative to percutaneous catheter drainage or surgical intervention.

\section{References}

1 Angulo JC, Dehaini A, Escribano J, SanchezChapado M: Successful conservative management of emphysematous pyelonephritis, bilateral or in a solitary kidney. Scand J Urol Nephrol 1997;31:193-197.

2 Shahatto N, Al Awadhi NZ, Ghazali S: Emphysematous pyelonephritis: surgical implications. Br J Urol 1990;66:572-574.

- 3 Lim CS, Kim WB, Kim YS, et al: Bilateral emphysematous pyelonephritis with perirenal abscess cured by conservative therapy. J Nephrol 2000;13:155-158.

-4 Tahir H, Thomas G, Sheerin N, Bettington H, Pattison JM, Goldsmith DJ: Successful medical treatment of acute bilateral emphysematous pyelonephritis. Am J Kidney Dis 2000;36:1267-1270.
5 Schenkman E, Auriemma P: Bilateral emphysematous pyelonephritis with autosomal dominant polycystic kidney disease. J Urol 1998;159:1633-1634.

- 6 Chou YH, Tiu CM, Chen TW, Chen KK, Chang T: Emphysematous pyelonephritis in a polycystic kidney: demonstration by ultrasound and computed tomography. J Ultrasound Med 1990;9:355-357.
7 Shimizu H, Hariu K, Kamiyama Y, Tomomasa H, Iizumi T, Yazaki T, Umeda T: Bilateral emphysematous pyelonephritis with autosomal-dominant polycystic kidney disease successfully treated by conservative method. Urol Int 1999;63:252-254.

$\checkmark 8$ Levinson ED, Weidner FA: Emphysematous pyelonephritis in a polycystic kidney. Urol Radiol 1981;3:39-41.

-9 Egawa S, Utsunomiya T, Uchida T, Mashimo S, Koshiba K: Emphysematous pyelonephritis, ureteritis, and cystitis in a diabetic patient. Urol Int 1994;52:176-178.

10 Huang JJ, Tseng CC: Emphysematous pyelonephritis: clinicoradiological classification, management, prognosis, and pathogenesis. Arch Intern Med 2000;160:797-805. 